\title{
An Input-Perceptual Reconstruction Adversarial Network for Paired Image-to-Image Conversion
}

\author{
Aamir Khan ${ }^{1}$, Weidong Jin ${ }^{1,2, *}$, Muqeet Ahmad ${ }^{3}$, Rizwan Ali Naqvi ${ }^{4}\left(\mathbb{D}\right.$ and Desheng Wang ${ }^{1}$ \\ 1 School of Electrical Engineering, Southwest Jiaotong University, Chengdu 611756, China; \\ aamir@my.swjtu.edu.cn (A.K.); wds@my.swjtu.edu.cn (D.W.) \\ 2 China-ASEAN International Joint Laboratory of Integrated Transport, Nanning University, \\ Nanning 530000, China \\ 3 School of Information Science and Technology, Southwest Jiaotong University, Chengdu 611756, China; \\ muqeetahmad@my.swjtu.edu.cn \\ 4 Department of Unmanned Vehicle Engineering, Sejong University, Seoul 05006, Korea; \\ rizwanali@sejong.ac.kr \\ * Correspondence: wdjin@home.swjtu.edu.cn
}

Received: 17 June 2020; Accepted: 23 July 2020; Published: 27 July 2020

\begin{abstract}
Image-to-image conversion based on deep learning techniques is a topic of interest in the fields of robotics and computer vision. A series of typical tasks, such as applying semantic labels to building photos, edges to photos, and raining to de-raining, can be seen as paired image-to-image conversion problems. In such problems, the image generation network learns from the information in the form of input images. The input images and the corresponding targeted images must share the same basic structure to perfectly generate target-oriented output images. However, the shared basic structure between paired images is not as ideal as assumed, which can significantly affect the output of the generating model. Therefore, we propose a novel Input-Perceptual and Reconstruction Adversarial Network (IP-RAN) as an all-purpose framework for imperfect paired image-to-image conversion problems. We demonstrate, through the experimental results, that our IP-RAN method significantly outperforms the current state-of-the-art techniques.
\end{abstract}

Keywords: image-to-image conversion; image de-raining; label to photos; edges to photos; generative adversarial network (GAN)

\section{Introduction}

The main objectives of image-to-image conversion tasks are the discovery of suitable latent space and understanding of features maps from source to target images. These tasks have multiple applications in computer graphics, image processing, and computer vision. Image processing applications include: (i) image in-painting, where damaged parts of an image are restored [1,2], (ii) image de-raining where rain-streaks are removed from an input image to get rain-free image [3,4], (iii) image super-resolution where high-quality images are generated from similar degraded images [5-10]. Additional applications exist, however they are not constrained to image denoising [11-13], style transfer [14], image segmentation [15] and image colorization [16,17].

Recently, researchers have developed convolutional neural networks (CNNs) for multiple image-to-image conversion problems. These models mostly come in the form of an encoder-decoder structure where the encoder encodes an input image to some latent space, the decoder decodes from the latent space to the required output image and then they punish the network with a loss function to pick up the mapping between two image domains. Many different loss functions and distinct motivations $[5,18]$ established these models. CNNs utilize reconstruction or pixel-wise 
losses $[5,17,19,20]$ to generate output images, which are the most upfront techniques. For example, in pixel space, the least absolute or the least-squares losses used to estimate the difference between the ground-truth and generated images. Pixel-wise computation can construct sensible photos. However, in many cases, these losses just capture low-frequency instead of high-frequency components of images, leading to some critical flaws concerning the outputs, e.g., image blurring and image artifacts [7].

Recent years have witnessed that the procedures using the concept of generative adversarial networks (GANs) [21] have accomplished remarkable results in image-to-image conversion tasks. GANs, introduced by Goodfellow et al., is made up of a generator network $G$ and a discriminator network $D$, targeting to model the real images distribution by synthesizing generated samples, which are very similar to real images. GAN-based models need more memory and computational time in the training process than simple $\mathrm{CNN}$ based models as they need to train two networks, i.e., the Discriminator network and the Generator network [22]. Whereas in the testing process, there is only one network, i.e., the generator network. Therefore, the memory and the computational time of GAN based models in the testing process are nearly similar to $\mathrm{CNN}$ based models. The significant advantage of using GAN based model is that it generates sharper and more realistic images than CNN based models [23-25]. Hence, the algorithms using the concepts of GANs and conditional GANs (cGANs) [26] have turned out to be a common approach for numerous image-to-image conversion tasks [8,23]. Based on cGAN, pix2pix-cGAN [23] became a representative method aimed at solving the paired image-to-image conversion problems, the objective of which is to map the conditional distribution of the real images conditioned on the given input images [25,27-29].

The critical part of image-to-image conversion tasks is that they have to map high-resolution input grids into high-resolution output grids. Additionally, the issue we consider is that the input and the output have dissimilar surface nature, but both must render the same basic structure to ensure perfect outcomes. There are two popular methods to find out the basic structure of an image, i.e., perceptual features based method [6] and moments based method [30]. The key challenges with methods of moments (MoM) [31] for training deep generative networks are in describing millions of sufficient distinct moments and identiflying an objective function for learning the desirable moments [31,32]. On the other hand, the use of features from deep neural networks (VGG-16) pre-trained on ImageNet dataset $[33,34]$ has led to important advancements in computer vision. Perceptual features have been widely used in piece of works such as super-resolution [6], style transfer [14], and transfer learning [35]. The image generation model is considered to learn from the information in the form of input images, which plays a significant part in the image-to-image conversion task to achieve desire targeted outputs. In paired image datasets, the input structure is roughly matched with the output structure and can significantly affect the production of the image generation models. For example, Figure 1 shows that the window frames are not accurately labeled in the corresponding input images. Hence, the image generation model requires further information to capture targeted high-resolution output grids against each given missing high-resolution input grids. Despite considerable progress, we note that the previous approaches have not examined optimized additional input information for imperfect paired datasets.

To overcome the problem of imperfect paired datasets and to attain desired results, we opted to feed this extra information in the form of input-perceptual loss (i.e., calculated between imperfect paired images) into the objective function of the proposed model. It is an essential issue, as the perfect paired dataset is expensive and hard to collect. This work introduces a trade-off between collecting large-amount of the perfect paired dataset and an optimized training for the image-to-image conversion network.

The remainder of the study is as follows: We discuss the previous research of the image-to-image conversion with details in Section 2. The IP-RAN methodology, objective, and network architecture are explained in Section 3. In Section 4, we present the experiments, results, and analysis of different loss functions and generator configurations. Section 5 presents the conclusions and future work. 


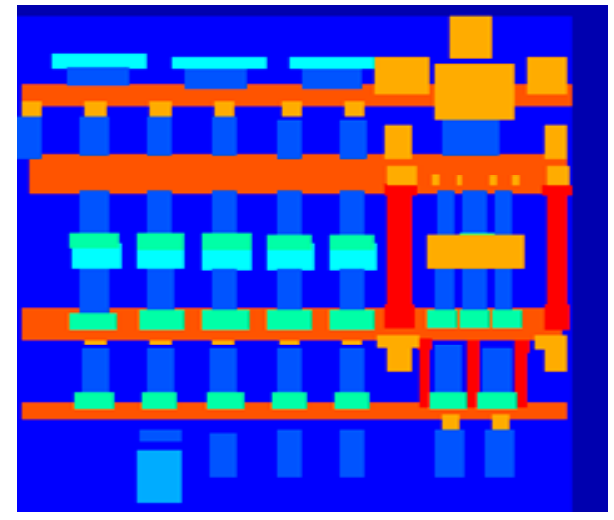

(a)

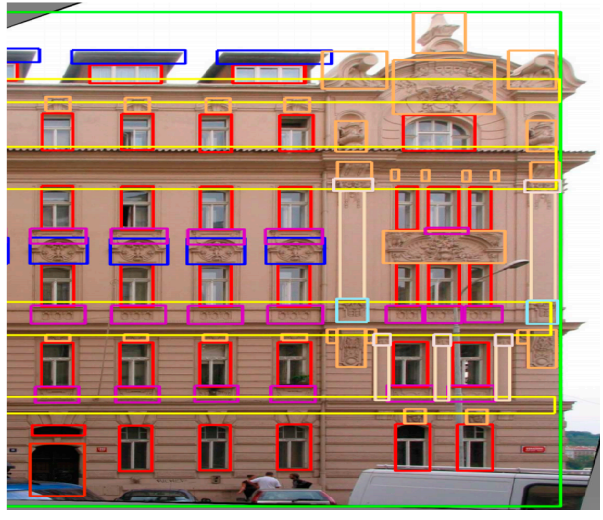

(b)

Figure 1. Example of the label to architectural photos. (a) shows an input labeled image. (b) shows marked objects in a ground-truth image.

\section{Related Work}

In previous years, the training of deep convolutional neural networks using back-propagation algorithms with per-pixel loss functions has solved a broad range of feed-forward image-to-image conversion tasks $[18,36]$. Various techniques of image-to-image conversion employ only pixel-level losses or pixel-level losses preceded by several additional losses [6,23]. Image segmentation techniques generate dense scene labels by operating networks in a fully convolutional way over a single input image [20,37-40]. Image de-raining techniques try to eliminate rain strikes in uncontrolled weather images [41,42]. Image super-resolution techniques generate a high-resolution image for a given its low-resolution matching part [5,6]. Image in-painting is designed to retrieve the missing portion of the given damaged image $[1,43,44]$. Other examples of image-to-image conversion techniques modeled on feed-forward CNNs exist, however, they are not constrained to depth estimations $[37,45,46]$ and image colorization [19], etc.

A series of GAN-family $[16,26,47,48]$ networks was introduced in a short time for an enormous variety of problems since Goodfellow introduced the influential concept of Generative Adversarial Nets (GAN) [21] in 2014. GANs also showed promising results in numerous applications for computer vision, for example, image generation, representation learning [48], image editing [49], etc. Specifically, various extended GANs accomplished good results at several image generation applications such as style transfer [24], super-resolution [7], image inpainting [1], text2image [50], and like many other domains including videos [51] and 3D data [52]. These studies also consist of but are not constrained to the PGN introduced for video prediction [53], the iGAN introduced for interactive application [54], the SRGAN added for super-resolution [7], and the ID-CGAN presented for image de-raining [3].

Moreover, some of these works based on GANs are dedicated to developing an improved generative model, for example, WGAN(-GP) [55,56], Energy-based GAN [57], Progressive GAN [58], SN-GAN [59] and E-GAN [60]. A conditional image generation based on GANs has also been actively studied recently. Some advanced GAN models continuously improved the quality of particular tasks, e.g., InfoGAN [16], cGANs [26], and LAPGAN [61] have been introduced to image translation recently for their easy execution and outstanding results. The cGANs [26] hold category labels as conditional data for the generation of particular images. Some of the works have included GANs into their designs to enhance the efficiency of conventional tasks, e.g., for small entity (or object) detection, the PGAN [62] was adopted. Specifically, Li et al. [62] developed an innovative perceptual-discriminator network, which includes a perception block and an adversarial block. Wang et al. [25] used different layers of discriminator network to measures perceptual losses. Sung et al. [63] introduced new paired input conditions for the replacement of conditional adversarial networks to improve the image-to-image translation tasks. 
Additionally, some modifications of the GANs [29,64-66] examined cross-domain image conversions over discovering the linear mapping relationship among various image domains. In particular, primal GAN intentions to investigate the mapping relationships between input images and target images, although a double (or opposite) GAN does the opposite task. Such GANs shape a closed-loop and enable the translation and reconstruction of images from either domain. These designs can also be used to execute image conversion operations in the lack of paired examples by merging cycle consistency loss and GAN loss. However, paired data is available for training in specific applications, Ge et al. [29], Zhu et al. [64], Yi et al. [65], and Kim et al. [66] ignore that paired data often achieves less than paired methods [23]. It is therefore still essential at this point to study paired data training, particularly for performance motivated circumstances and implementations like the photo-realistic picture synthesis [7], high-resolution image synthesis [8], real-world image painting [67], etc.

In GANs based works, generator networks are the same as the aforementioned encoder-decoder structure in CNNs. As the training of deep CNNs suffer from vanishing gradient problem. Therefore, many previous works $[3,4,25]$ used skip-connections in the generator to pass the gradient easily to prior layers of the encoder. Unfortunately, these skip-connections directly carry unwanted information from the inputs to the resultant images, hence affecting the visual quality of the constructing images. In the demand to develop a visually appealing image-to-image conversion model, we have to consider the following facts into the optimization method:

- The principle, to perfectly map targeted output images must not be affected by the texture of the given input images, which should be the essential pillar in the formation of a generator structure.

- The visual quality of constructed images should also be considered in the optimization method rather than just relying on qualitative performance metric values. This principle can guarantee that the generated images look visually appealing and realistic.

Under the above criteria, we present the Input-Perceptual and Reconstruction Adversarial Networks (IP-RAN) for image-to-image conversion tasks. The IP-RAN consists of an encoder-decoder network $G$; for converting an input image to the desired output image, a discriminator network $D$; to flag the real or fake photos and an input-perceptual loss network $P$; to calculate fundamental structure difference between an input image and the ground-truth image. We employe the input-perceptual, the traditional reconstruction L1, and the generative adversarial losses in the objective function. Initially, this work utilized the input-perceptual loss to calculate the missing information of the basic structure in the input images according to the target images. Then, this study used similar to many traditional losses the L1 loss for penalizing generated images to be near to the targeted images. Meanwhile, we used the generative adversarial losses to estimate the distribution of converted images, i.e., to punish the generated distribution for converging into the target distribution of output, which generally results in the production of more visually pleasing images. The contributions of this study are as follows:

- This study introduces a novel approach to deal with imperfect paired datasets and the method of feeding extra information into the objective function in the form of input-perceptual losses calculated between the input images and the target images for imperfect paired datasets.

- We introduce an optimized method based on pix2pix-cGAN and conditional GANs (cGANs) frameworks for existing imperfect pair datasets.

- We also analyzed the primary two different configurations of the generator structure, and the results show the proposed approach is better than previous methods.

- We achieve both qualitative and quantitative results by using IP-RAN, which indicates that the adopted technique produces better results than the baseline models.

Table 1 shows a comparison between the proposed and existing methods. 
Table 1. Comparison between state-of-the-art and proposed method.

\begin{tabular}{|c|c|c|}
\hline Methods & Advantages & Disadvantages \\
\hline \multirow{2}{*}{$\begin{array}{l}\text { CNNs (Reconstruction L1 and L2 } \\
\text { losses) based methods }[1,17]\end{array}$} & $\begin{array}{l}\text { Need less computation as one } \\
\text { network is to trained. }\end{array}$ & Need big datasets to train \\
\hline & Fast and easy to train & Produce blurry results \\
\hline \multirow{3}{*}{$\begin{array}{l}\text { Simple GAN (Adversarial Loss) } \\
\text { based methods }[21,26]\end{array}$} & Can be trained with small datasets & $\begin{array}{c}\text { More computation than } \mathrm{CNNs} \text { as } \\
\text { two different networks } \\
\text { to be trained }\end{array}$ \\
\hline & \multirow{2}{*}{$\begin{array}{l}\text { Produces sharp and } \\
\text { realistic images }\end{array}$} & GAN networks are difficult to train \\
\hline & & There is an image artifacts problem \\
\hline \multirow{2}{*}{$\begin{array}{l}\text { Adversarial, reconstruction and } \\
\text { perceptual losses with } \\
\text { skip-connections in generator } \\
\text { network based methods } \\
{[3,4,23,25,29,63]}\end{array}$} & $\begin{array}{l}\text { Achieve good quality results than } \\
\text { CNNs and simple GAN by } \\
\text { combining two loss functions }\end{array}$ & \multirow{2}{*}{$\begin{array}{l}\text { Skip-connections affect the quality } \\
\text { of generated images by directly } \\
\text { passing unwanted input } \\
\text { information to the output } \\
\text { of the network. }\end{array}$} \\
\hline & $\begin{array}{c}\text { Skip-connections in generator } \\
\text { configuration reduce vanishing } \\
\text { gradient problem }\end{array}$ & \\
\hline \multirow{3}{*}{ Proposed method } & $\begin{array}{l}\text { This method adds extra } \\
\text { information to the objective } \\
\text { function to optimize the results. }\end{array}$ & \multirow{3}{*}{$\begin{array}{l}\text { Need to calculate input-perceptual } \\
\text { losses which increase training time }\end{array}$} \\
\hline & $\begin{array}{l}\text { Use the Resnet bottleneck } \\
\text { structure in the generator } \\
\text { configuration to reduce the } \\
\text { vanishing gradient problem. }\end{array}$ & \\
\hline & $\begin{array}{l}\text { Achieves excellent results visually } \\
\text { and quantitatively }\end{array}$ & \\
\hline
\end{tabular}

\section{Methodology}

In this work, we have two sets of paired training images, i.e., a set of input images $\left\{x_{i}\right\}_{i=1}^{N} \in X$ and a set of target output images $\left\{y_{i}\right\}_{i=1}^{N} \in Y$. We train the generative network $G$ that the fake generated images $G(x)$ to be same as the real targeted images, and alongside we train a discriminative network $D$ to distinguish the fake generated images $G(x)$ from the real targeted images. The generator network learns the mapping from an input domain to a real-world domain by minimizing adversarial losses, aiming to deceive the discriminator network. The generator has sub-networks: an encoder $E n c$, residual blocks Res, and a decoder network Dec. The encoder network contains a sequence of convolutional layers, which convert an input image into encoded feature space $\operatorname{Enc}(x)$. Later, the output of encoder network, Enc $(x)$, becomes the input of residual blocks [68]. The output of the residual layers, $\operatorname{Res}(\operatorname{Enc}(x))$, is the activation maps which feed to the decoder network Dec. At that moment, a sequence of fractionally-stride convolutionary layers decode the converted features into the fake generated image $G(x)$. Equation (1) expresses the output of the generator network:

$$
G(x)=\operatorname{Dec}(\operatorname{Res}(\operatorname{Enc}(x)))
$$

The whole network architecture is shown in Figure 2 and is called the Input-Perceptual Pixel-Reconstruction Generative Adversarial Networks (IP-RAN). 


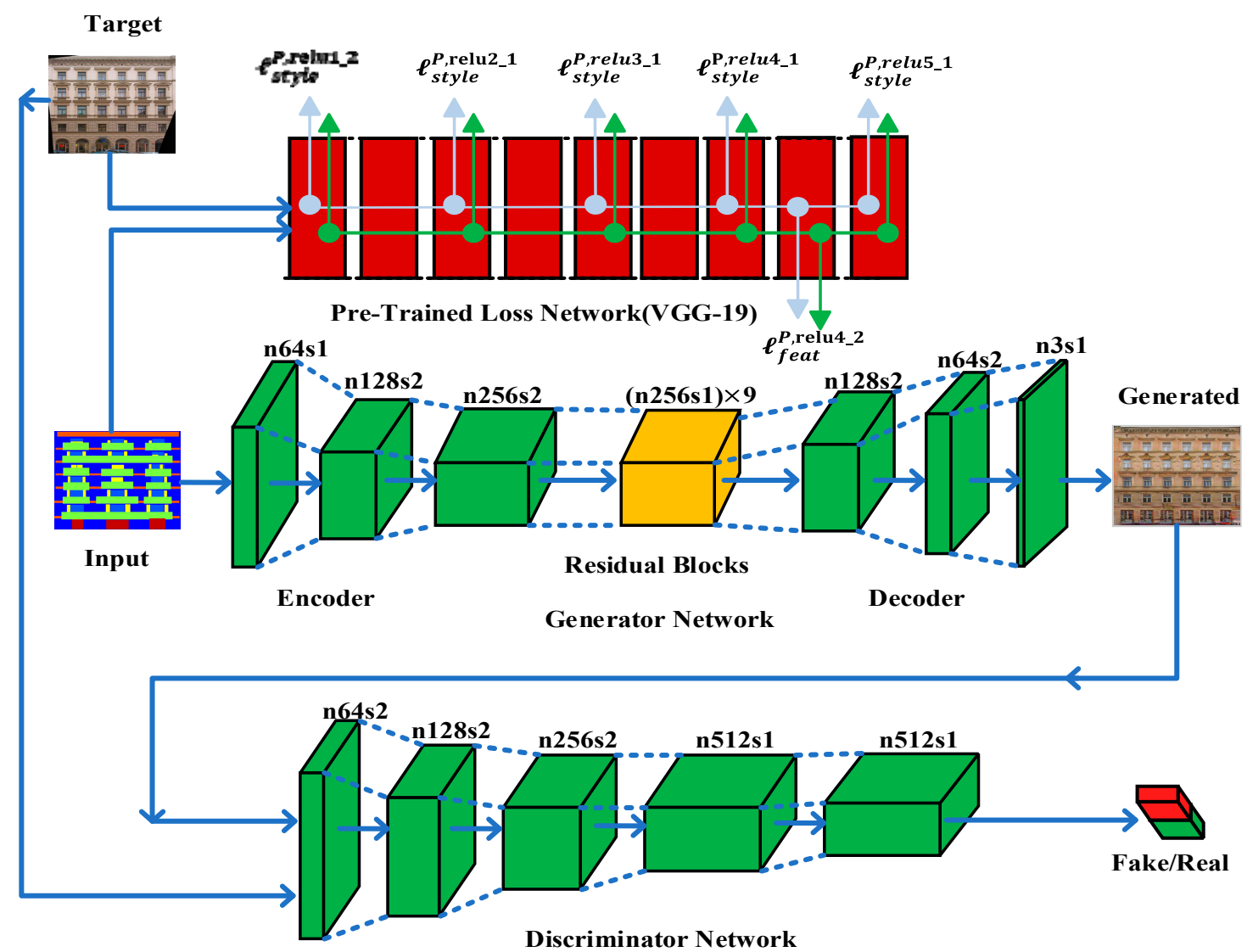

Figure 2. IP-RAN framework. IP-RAN consists of generator network, $G$, input-perceptual loss network, $P$, and discriminator network, $D$. The generator network, $G$, is intended to generate translated images from given input images. It is composed of an encoder-decoder structure that includes two down-sampling layers of stride- 2 convolution, several residual blocks, and two up-sampling layers stride-2 of transposed convolution. Input-perceptual loss network, $P$, is the pre-trained VGG-19 and used to extract features from hidden layers to calculate the perceptual loss. The discriminator network, $D$, consists of convolutional-BatchNorm-LeakyRelu layers, and its output is used to distinguish generated images from real images.

\subsection{Objective}

The input-perceptual loss calculated between high-resolution input grids and targeted high-resolution output grids, which decrease the effect of less information in the input images and useful against imperfect paired datasets. Equation (2) expresses input-perceptual loss:

$$
\mathcal{L}_{P}(P)=\varphi_{c} l_{f}+\varphi_{s} l_{s}
$$

where $l_{f}$ is the feature reconstruction as given in Equation (3), and $l_{s}$ is the style reconstruction losses as given in Equation (4), are the two parts of the perceptual loss function, as Johnson et al. described in [6]. Input-perceptual losses are utilized to measure fundamental structural differences such as common patterns, texture, colors, etc., between the high-resolution input grids and the high-resolution target grids.

Let $P_{i}(x)$ be the activation maps for the $i^{\text {th }}$ layer of the network $P$ when processing the image $x$. If $i$ is a convolutional layer then $P_{i}(\mathrm{x})$ will be an activation map having a shape of $C_{i} \times H_{i} \times W_{i}$. The feature reconstruction loss can be calculated as Euclidean distance between activation maps as follows:

$$
l_{f}=\ell_{\text {feat }}^{P, i}(x, y)=\frac{1}{C_{i} H_{i} W_{i}}\left\|P_{i}(x)-P_{i}(y)\right\|_{2}^{2}
$$


where $P_{i}$ denotes the non-linear CNN transformation at the $i^{\text {th }}$ layers of the loss network, $P$. The $\ell_{\text {feat }}^{P}$ loss aims to measure the discrepancy between high-level features of the given images.

The style reconstruction loss can be computed as squared Frobenius norm for the discrepancy between the Gram matrices of the input and the targeted images as follows:

$$
l_{s}=\ell_{\text {style }}^{P, i}(x, y)=\left\|\mathbb{G}_{i}^{P}(x)-\mathbb{G}_{i}^{P}(y)\right\|_{F}^{2}
$$

where $\mathbb{G}_{i}^{P}(x)$ is the Gram matrix of $i^{t h}$ layer activation maps of a given image $x$ extracted from network $P$. $\mathbb{G}_{i}^{P}(x)$ is defined as the components of the $C_{i} \times C_{i}$ matrix is given by:

$$
\mathbb{G}_{i}^{P}(x)_{c, c^{*}}=\frac{1}{C_{i} H_{i} W_{i}} \sum_{h=1}^{H_{i}} \sum_{w=1}^{W_{i}} P_{i}(x)_{h, w, c} P_{i}(x)_{h, w, c^{*}}
$$

where $P_{i}(x)$ interpret as giving $C_{i}$-dimensional activation maps for each point on $H_{i} \times W_{i}$ grid, and the Gram matrix, $\mathbb{G}_{i}^{P}(x)$, relates to non-centric covariance of the $C_{i}$-dimensional activation maps, processing each grid site as an autonomous sample. Therefore, it gathers details about the features that appear to be working together. The Gram matrix can also be determined accurately by transforming $P_{i}(x)$ into a matrix $\phi$ of shape $C_{i} \times H_{i} W_{i}$; then $\mathbb{G}_{i}^{P}(x)=\frac{\phi \phi^{T}}{C_{i} H_{i} W_{i}}$.

Generative adversarial loss [21], which trains $G$ and $D$ together as the two-player mini-max game with loss function $\mathcal{L}_{G A N}(G, D)$. The generator network $G$ attempts to produce an image $G(x)$ that appears similar to the image in the target domain $Y$, while the discriminator network $D$ attempts to differentiate between them. In particular, we train the discriminator network, $D$, to maximize the likelihood of classifying the correct label to the targeted image and the generated image $G(x)$, while training $G$ is to minimize the likelihood of classifying the correct label to the generated image $G(x)$. The mini-max game can be formulated as:

$$
\min _{G} \max _{D} \mathbb{E}_{y \in Y}[\log (D(y))]+\mathbb{E}_{x \in X}[\log (1-D(G(x)))]
$$

GANs-based models have revealed the significant ability to learn generative models, particularly for image generation tasks $[16,53,55]$. Therefore, we also implement the GANs learning process to resolve image conversion tasks. As illustrated in Figure 2, the image generation network $G$ is used to produce output image, $G(x)$, against the input image, $x \in X$. In the meantime, each input image $x_{i}$ has a correspondent target image $y_{i}$. We assume that all target images, $y$, follow the distribution $y \in Y$, and the generated images, $G(x)$, are motivated to have matching distribution as targeted images $y$, i.e., $G(x) \sim Y$. Besides, to accomplish the generative adversarial learning approach, a discriminative network, $D$, is added, and the adversarial loss function can be expressed as follows:

$$
\min _{G} \max _{D} V(G, D)=\mathbb{E}_{y \in Y}[\log (D(y))]+\mathbb{E}_{x \in X}[\log (1-D(G(x)))]
$$

We use least squares loss (LSGAN) as discussed in [69], which offers a non-saturated and smooth gradient for discriminator network $D$. Adversarial loss, $\mathcal{L}_{G A N}(G, D)$, is expressed as:

$$
\mathcal{L}_{G A N}(G, D)=\mathbb{E}_{y \in Y}\left[(D(y)-1)^{2}\right]+\mathbb{E}_{x \in X}\left[D(G(x))^{2}\right]
$$

The generative adversarial loss turns as per the numerical measurement to punish the variance between the distributions of generated images and ground-truth images.

The basic GAN framework is unstable as it trains two competing neural networks. In [64], the author noted that one cause for instability is that there are un-unique solutions during the training of the generator. As shown in Figure 3, several artifacts introduced by the standard GAN structure can be observed which significantly impacts the visual quality of the output image. Previous methods 
have found that it is useful to combine GAN objectives with more traditional losses such as L2 loss [1] in such way that the work of the discriminator remains unchanged as in Equation (8), but the task of the generator is not only to deceive the discriminator but also to make generated image closer to the targeted ground truth image according to L2. In our method, we used L1 distance instead of L2, because L1 encourages blur reduction:

$$
\mathcal{L}_{L 1}(G)=\mathbb{E}_{x, y}\left[\|y-G(x)\|_{1}\right]
$$

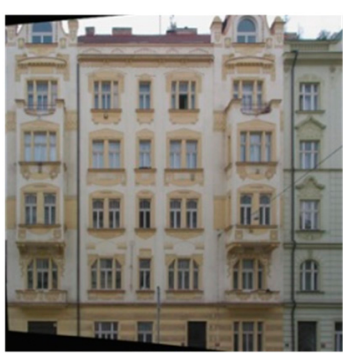

(a)

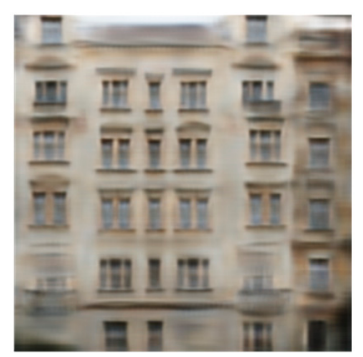

(b)

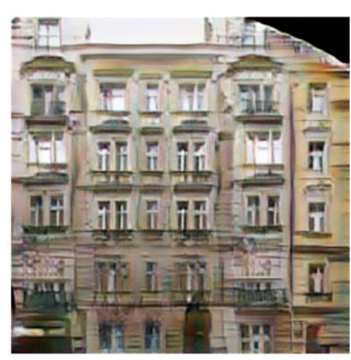

(c)

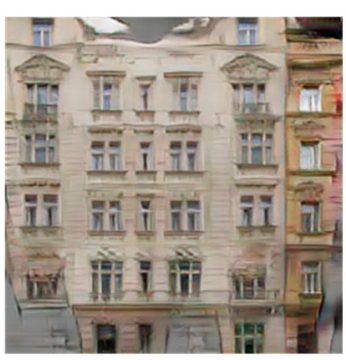

(d)

Figure 3. (a) ground-truth image, (b) image generated by conventional CNN using L1 loss function, (c) image generated by standard GAN using adversarial loss and (d) image constructed by the proposed method with Input-Perceptual and Reconstruction Adversarial losses

The adversarial loss helps the generator and protect from the blurry effect of L1 loss as well as remain close to the targeted output images. The final objective for the generator network is expressed as:

$$
\mathcal{L}_{G_{T}}=\varphi_{g} \mathcal{L}_{G A N}(G)+\varphi_{L 1} \mathcal{L}_{L 1}(G)+\varphi_{P} \mathcal{L}_{P}(P)
$$

where $\mathcal{L}_{G_{T}}$ represents the total generator network loss which is the sum of the generator's adversarial loss, $\mathcal{L}_{G A N}(G)$, L1 reconstruction loss, $\mathcal{L}_{L 1}(G)$, and the input-perceptual, $\mathcal{L}_{P}(P)$.

\subsection{Network Architecture}

Figure 2 demonstrates the proposed structure consisting of three CNNs networks, i.e., the generator network, $G$, the input-perceptual loss network, $P$, and the discriminative network, $D$.

Recently, many solutions $[3,23,25]$ to these problems used skip-connections in the generator network to shuttle the information directly from input to output throughout the network and to solve the vanishing gradient problem. On the one hand, skip-connections are useful in resolving the vanishing gradient problem. Still, for image-to-image conversion problems, these skip-connections are carrying unwanted information from the input throughout the network and influencing the performance of the results critically, see Figure 2. We utilize the ResNet [68] framework same as Johnson et al. [6], with an encoder-decoder structure instead of skip-connections between encoder-decoder layers to avoid unwanted information coming from the input and to produce visually pleasing results. Our generator network includes two downsampling layers of stride-2 convolution, nine residual blocks, and two upsampling layers with stride-2 of transposed convolution and utilizes instance normalization [70], for specifications, see Table 2. The input-perceptual loss network, $P$, uses VGG-19 pre-trained on the ImageNet dataset [33,34]. We extract features from six layers (Relu-1 of block1, Relu-1 of block2, Relu-1 of block3, Relu-1 of block4, Relu-1 of block5) for style loss $l_{s}$ and Relu-2 of block4 for feature loss $l_{f}$ of pre-trained VGG-19 to calculate input-perceptual losses.

In this work, we use $70 \times 70$ Markovian PatchGANs $[7,23,71]$ for the discriminator network $D$ to classify whether $70 \times 70$ overlapping patches of images are real or fake. Patch-level discriminator has fewer parameters than a full-image discriminator and can operate in a fully convolutionary fashion on images of arbitrary size [23]. 
Table 2. Generator Network of IP-RAN.

\begin{tabular}{|c|c|c|c|c|c|c|}
\hline & Operation & $\begin{array}{l}\text { Pre-Reflection } \\
\text { Padding }\end{array}$ & Kernel Size & Stride & Non-Linearity & Feature Maps \\
\hline \multirow{3}{*}{$\begin{array}{c}\text { Encoder } \\
\text { entry } 2\end{array}$} & Convolution & 3 & 7 & 1 & ReLU & 64 \\
\hline & Convolution & & 3 & 2 & ReLU & 128 \\
\hline & Convolution & & 3 & 2 & ReLU & 256 \\
\hline \multirow{9}{*}{$\begin{array}{l}\text { Residual } \\
\text { Blocks }\end{array}$} & Residual block & 1 & 3 & 1 & ReLU & 256 \\
\hline & Residual block & 1 & 3 & 1 & ReLU & 256 \\
\hline & Residual block & 1 & 3 & 1 & ReLU & 256 \\
\hline & Residual block & 1 & 3 & 1 & ReLU & 256 \\
\hline & Residual block & 1 & 3 & 1 & ReLU & 256 \\
\hline & Residual block & 1 & 3 & 1 & ReLU & 256 \\
\hline & Residual block & 1 & 3 & 1 & ReLU & 256 \\
\hline & Residual block & 1 & 3 & 1 & ReLU & 256 \\
\hline & Residual block & 1 & 3 & 1 & ReLU & 256 \\
\hline \multirow{3}{*}{ Decoder } & Deconvolutional & & 3 & 2 & ReLU & 128 \\
\hline & Deconvolutional & & 3 & 2 & ReLU & 256 \\
\hline & Convolutional & 3 & 7 & 1 & Tanh & 256 \\
\hline
\end{tabular}

\section{Experiments and Results}

In this section, we first discuss the specifications of the datasets, proposed model, and training parameters. We compared the IP-RAN with the standard approaches and current state-of-the-art methods. We also discuss the information on the experiments and performance measures used to test the proposed method.

\subsection{Datasets}

Experiments are carried out on several datasets to evaluate the performance of IP-RAN and other state-of-the-art methods. We use three public paired datasets which are as follows:

- CMP facades dataset [72] is used to train for architectural "Labels to Photos" task.

- Dataset provided by ID-CGAN [3] is used to train for the "Image De-raining" task.

- Dataset formed by pix2pix [23] is used to train for the "Edges to Photos" task. The original dataset has come from [54] and [73], and the use of the HED edge detector [74] to extract edges. All images are scaled to $256 \times 256$.

\subsection{Model and Parameter Details}

In this subsection, we discuss the model and the parameter details. In the case of GAN loss $\left(\mathcal{L}_{G A N}\right)$, we replace the criterion of negative log-likelihood with a least-square loss [69] for the network's training stabilization. This least-square loss is found more stable throughout the training procedure and produces higher quality results. In general, for $\mathcal{L}_{G A N}(G, D)$, we set that $G$, train to minimize $E_{x \sim p_{\text {data }}(x)}\left[(D(G(x))-1)^{2}\right]$ and $D$, train to minimize $E_{y \sim p_{\text {data }}(y)}\left[(D(y)-1)^{2}\right]+E_{x \sim p_{\text {data }}(x)}\left[\left(D(G(x))^{2}\right]\right.$. Furthermore, we divide the discriminator's criterion by 2 when optimizing $D$, which slows the learning rate of $D$ proportional to $G$. We apply the Adam optimizer [75] and use minibatch Stochastic Gradient Decent (SGD), setting a learning rate of $\alpha=0.0002, \beta 1=0.5$. Relu activation function, with slope value of 0.2 , is used in the generator network, $G$, except the last layer used tanh. The Batch size is set to one for all of the experiments. The training parameters are set as $\varphi_{g}=1, \varphi_{L 1}=10, \varphi_{s}=1$ and $\varphi_{c}=0$ for labels to photos task, $\varphi_{g}=1, \varphi_{L 1}=10, \varphi_{s}=1$ and $\varphi_{c}=1 \times 10^{-6}$ for edges to photos task, and $\varphi_{g}=1 \times 10^{-9}, \varphi_{L 1}=10, \varphi_{s}=1$ and $\varphi_{c}=1 \times 10^{-6}$ for image de-raining task. 


\subsection{Evaluation Criteria}

For a performance demonstration of image-to-image conversion tasks, we performed qualitative and quantitative tests to determine the quality of the generated images. We directly present input and generated images for qualitative assessments. We apply quantitative measures on test sets to assess the performance of different model and configurations such as, Peak Signal to Noise Ratio (PSNR), Structural Similarity Index (SSIM) [76], Visual Information Fidelity (VIF) [77], and Universal Quality Index (UQI) [78]. These quantitative measures valuation are based on the luminance channel of the image. FID score [79] determines the distance between the real data distribution and the generated data distribution.

\subsection{Analysis of Different Loss Functions}

We train models to separate the effect of different variations of loss functions on the architectural CMP facades "label to photos" dataset. We perform tests to compare the impact of each part of Equation (10). Figure 4 shows the qualitative results of the variations mentioned below on labels to photos problem.

- $\quad$ L1, by setting $\varphi_{g}=0$ and $\varphi_{P}=0$ in Equation (10), causes to generate blurry outputs.

- The cGAN, by setting $\varphi_{L 1}=0$ and $\varphi_{P}=0$ in Equation (10), leads to much sharper outputs but brings visual artifacts.

- $\quad$ L1 and cGAN together, by setting $\varphi_{P}=0$ in Equation (10) causes sensible results but still far from the targeted outputs.

- The results of the proposed loss function in Equation (10), show a significant improvement in quality and similarity to the targeted results.

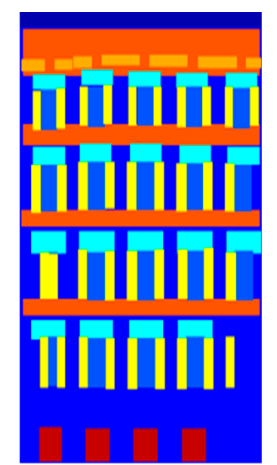

(a)

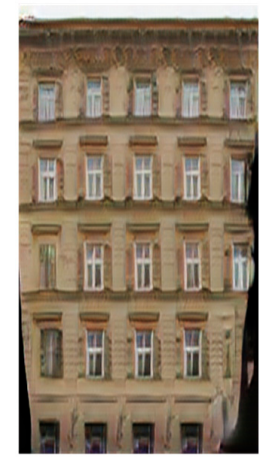

(d)

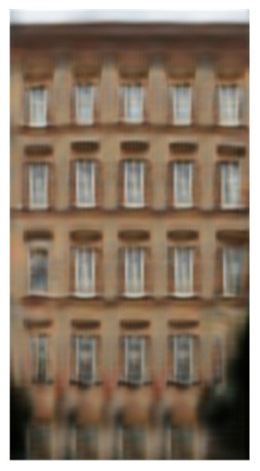

(b)

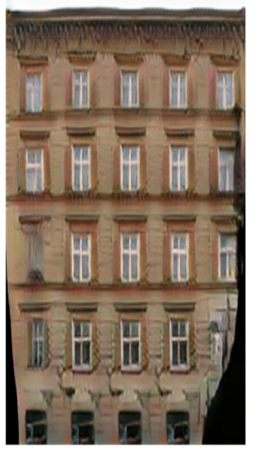

(e)

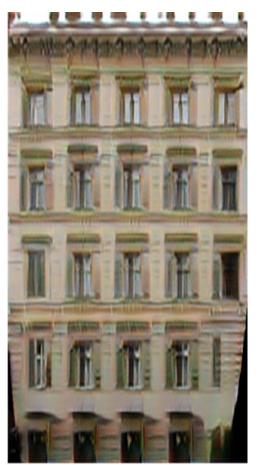

(c)

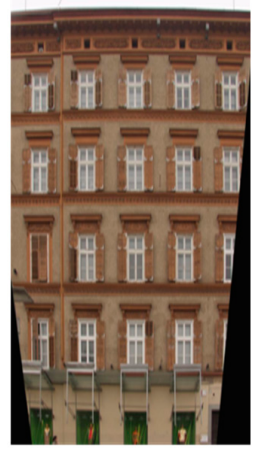

(f)

Figure 4. Shows label input against different loss functions that produce different architectural photo results. (a) input label image, (b) result of L1 $\left(\mathcal{L}_{L 1}(G)\right)$ alone, (c) result of cGAN $\left(\mathcal{L}_{G A N}(G)\right)$ alone, (d) result of L1+cGAN $\left(\mathcal{L}_{G A N}(G)+\mathcal{L}_{L 1}(G)\right)$, (e) result of the IP-RAN, and (f) target output photo. 
In Table 3, we compared the abovementioned cases quantitatively using the PSNR, SSIM, UQI, VIF, and FID scores on the labels to photos dataset. L1 achieves higher scores in PSNR, SSIM, UQI, and VIF, but the output results are blurred images and are very poor in FID-score. Hence, pointing out that the results are visually unpleasant. We observed from Figure 4 and Table 3 that for blurry images PSNR, SSIM, UQI, and VIF evaluation scores perform inferiorly. Table 3 shows that cGAN alone achieves poor scores in PSNR, SSIM, UQI, and VIF, which indicating that results are less similar to the targeted output. However, it has got a good FID-score as compare to L1 that shows results have a recognizable structure. Table 3 shows that the IP-RAN achieves the best possible scores in PSNR, SSIM, UQI, VIF, and FID. Hence, the results are similar to the targeted output as well as have a recognizable structure, and they are visually pleasing.

Table 3. Quantitative results compared with different loss functions.

\begin{tabular}{cccccc}
\hline & PSNR(dB) & SSIM & UQI & VIF & FID \\
\hline L1 & $\mathbf{1 3 . 4 3}$ & $\mathbf{0 . 2 8 3 7}$ & $\mathbf{0 . 8 1 8 6}$ & $\mathbf{0 . 0 6 2 7}$ & 176.74 \\
cGAN & 11.86 & 0.1996 & 0.7722 & 0.0399 & 111.00 \\
L1+cGAN=CGAN & 12.80 & 0.2399 & 0.8035 & 0.0480 & 113.53 \\
IP-RAN & 12.84 & 0.2426 & 0.8052 & 0.0488 & $\mathbf{1 1 0 . 2 9}$ \\
\hline
\end{tabular}

\subsection{Analysis of Different Generator Configuration}

The encoder-decoder structure does not have skip-connections among the layers. The U-Net structure has skip-connections between encoder layers and decoder layers, as shown in Figure 5. We have trained both structures on image de-raining dataset and labels to photos dataset with similar loss function using pix2pix-cGANs [23] architecture. We conducted tests to compare both structures.

\section{Encoder-Decoder}

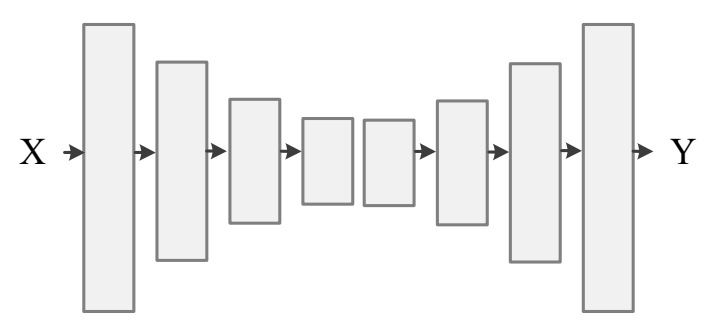

\section{U-NET with Skip-Connections}

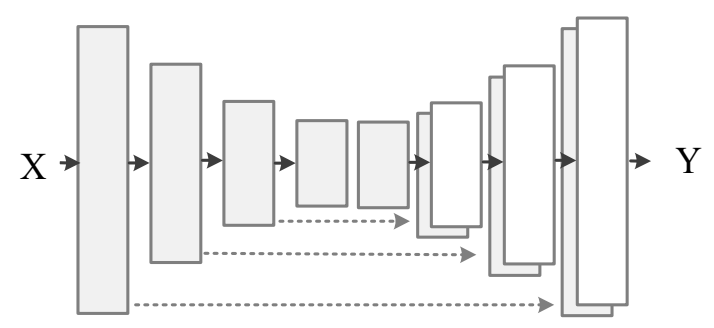

Figure 5. Different structures of image-to-image generation networks.

Figure 6 shows the encoder-decoder structure achieves excellent results without losing any information than the U-Net structure. Skip-connections passing unwanted information of the input images, which have a severe influence on generated images, leads to corrupted results and poorly achieved their targets. In the image de-raining task, the generator structure with skip-connections poorly converts between the rain to de-raining images. In Figure $6 c$ the first four rows, where rain-streaks still can be found in resultant images. The resultant images inherit this unwanted information via skip-connections from the corresponding input images. Figure 6c the last four rows, where resultant images contain bluish and greenish color effects, which are directly coming from the input labeled images via skip-connections. 

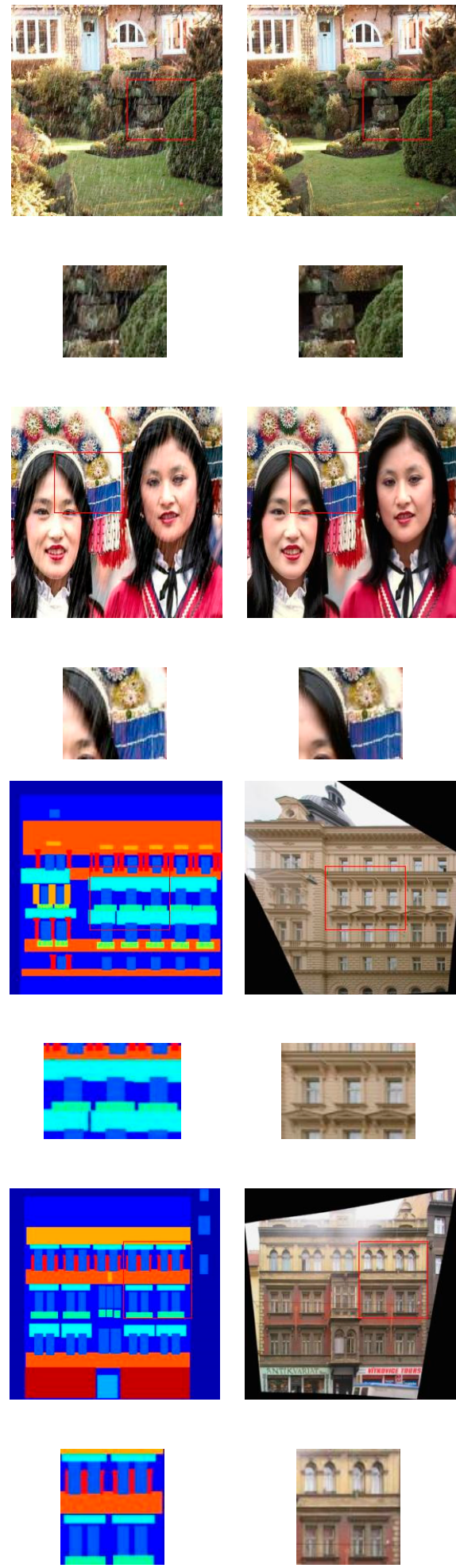

(a)

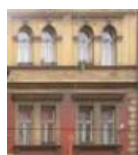

(b)
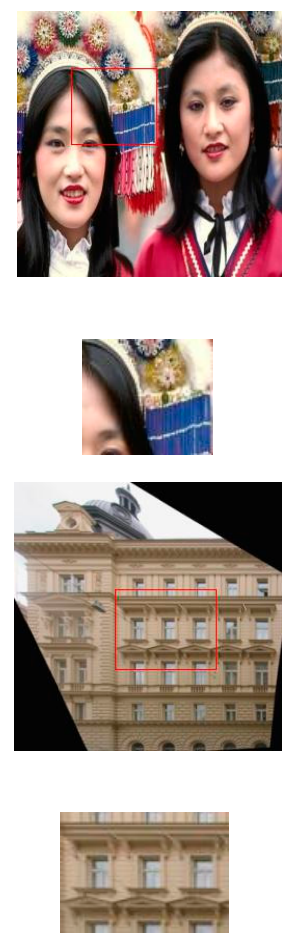
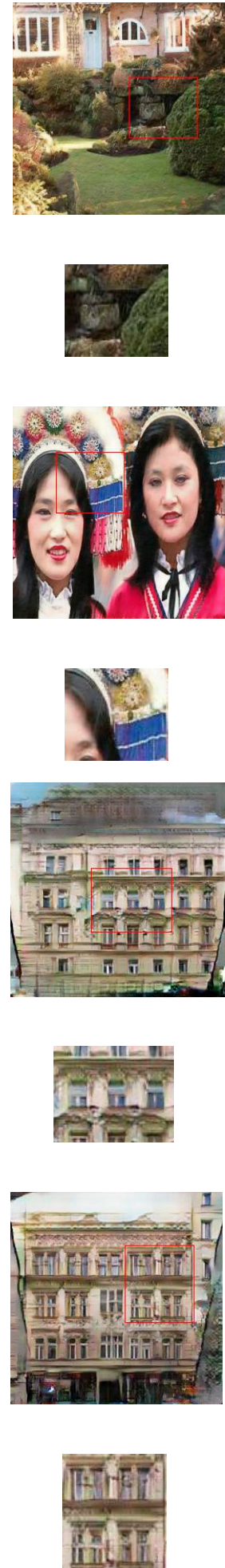

(c)
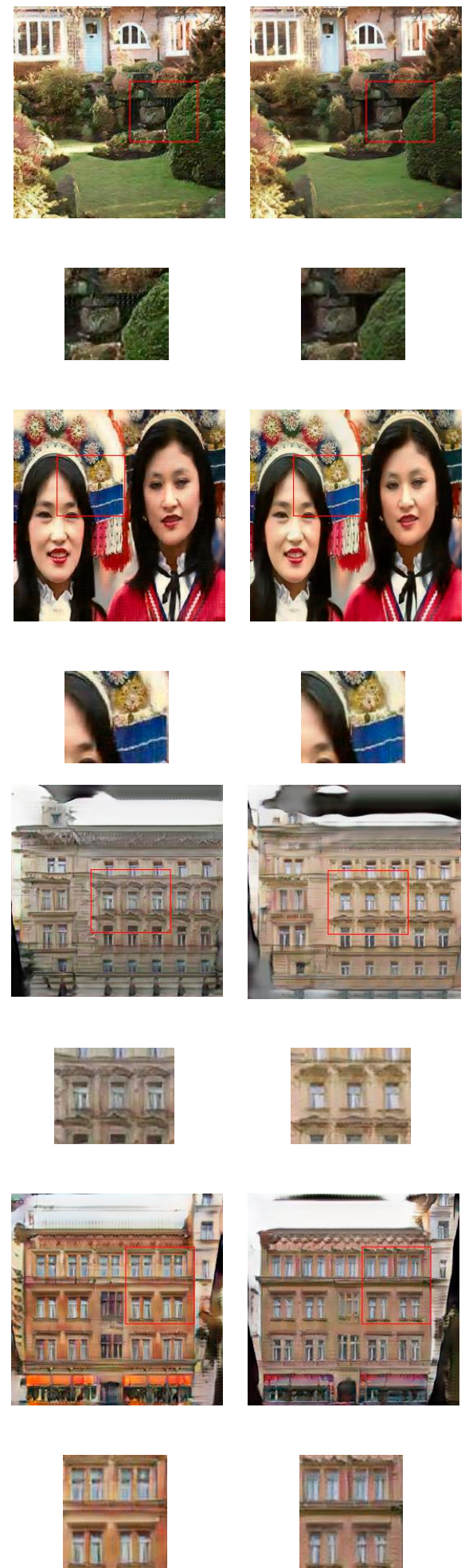

(d)

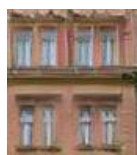

(e)

Figure 6. Sample results in the first four rows of rainy to de-raining images and last four rows of labels to architectural photos. For good visual comparison, the smaller images below the test images represent specific regions-of-interest. (a) input images, (b) targeted photos, (c) U-Net with skip-connections, (d) Encoder-Decoder and (e) IP-RAN. 


\subsection{Comparison with Baseline}

For comparison purposes, we selected the following latest state-of-the-art approaches for image-to-image conversion problems:

- Pix2Pix-cGAN [23]: Pix2pix is designed for paired image datasets based on the cGAN architecture. Pix2Pix utilizes L1 reconstruction loss and adversarial loss to train its model for the conversion of input images to output images.

- UTN-GAN [29]: UTN-GAN introduced a GAN-based unsupervised transformation network with hierarchical representations learning and weight-sharing technique. The reconstruction network learns the hierarchical representations of the input image, and the mutual high-level representations are shared with the translation network to realize the target-domain oriented image translation.

- $\quad$ PAN [25]: PAN can learn a mapping function to transform input images to targeted output images. PAN consists of a image transformer network and a discriminator network. In PAN, the discriminator measures perceptual losses on different layers and identifies between real and fake images. PAN uses perceptual adversarial losses to train the generator model.

- $\quad$ iPANs [63]: iPANs used U-NET as image transformation network and perceptual similarity network as a discriminator network. iPANs introduced new paired input conditions for the replacement of conditional adversarial networks to improve the image-to-image translation tasks. In this method the ground-truth images which are identical images are the real pair, whereas the generated images and ground-truth images are the fake pair.

- ID-CGAN [3]: ID-CGAN introduced to handle the image de-raining task by combining the pixel-wise least-squares reconstruction loss, conditional generative adversarial losses, and perceptual losses. ID-CGAN used cGAN structure to map from rainy images to de-rainy images. ID-CGAN consists of a dense generator to transform from an input image to its counter-part output images. ID-CGAN used the pre-trained VGG-16 network to calculate the perceptual losses between generated and ground-truth images.

\subsubsection{Comparison with Pix2Pix-cGAN, PAN, UTN-GAN and iPANs}

We attempt to transform semantic labels to architectural photos. This inverse conversion is a complicated process and distinct from the tasks of image segmentation. Pix2Pix-cGAN and UTN-GAN used adversarial and reconstruction losses, and PAN and iPANs used adversarial and perceptual losses to produce labels to architectural photos as shown in Figure 7. After the comparison, we observe the adopted approach captures further information and generates realistic and more similar images to the targeted photos with less deformation. Furthermore, the quantitative assessment in Table 4 also demonstrates that the IP-RAN can attain substantially improved results.

Creating a real-world object from the corresponding input edges is one of the image-to-image conversion tasks as well. We train the IP-RAN on the dataset given by [23] to convert edges-to-shoes and compare its results by the outcomes of pix2pix-cGAN, PAN, UTN-GAN and iPANs. Figure 8 shows shoe photos generated from given input edges by the proposed method, pix2pix-cGAN, PAN, UTN-GAN and iPANs, while Table 5 presents the quantitative measures on the test set results. By observing and comparing the constructed shoe photos, we find that the IP-RAN, pix2pix-cGAN and PAN accomplished promising results, so far, it's difficult to express which of these is better. On the measurement score of UQI and FID, the IP-RAN performed slightly weak compared to pix2pix-cGAN and PAN, yet superior in the other quantitative measures. 

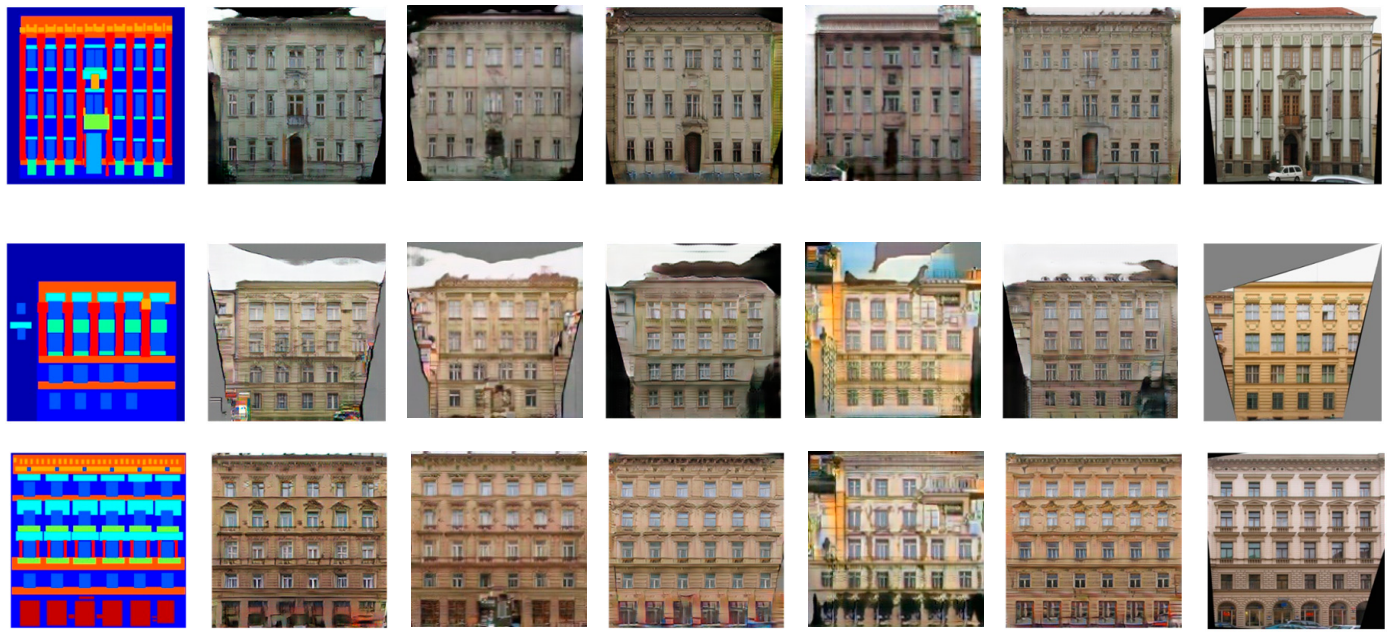

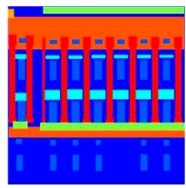

(a)

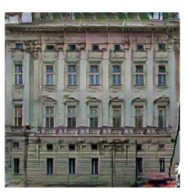

(b)

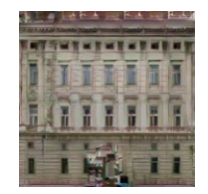

(c)

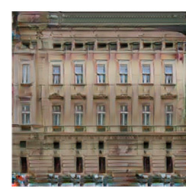

(d)

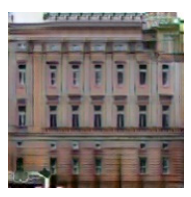

(e)

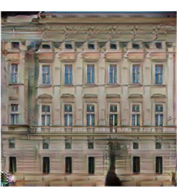

(f)

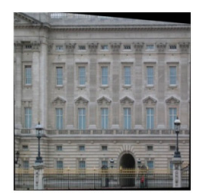

(g)

Figure 7. Samples results from paired labels to architectural photos. (a) input images, (b) results of pix2pix-cGAN, (c) results of UTN-GAN, (d) results of PAN, (e) results of iPANs, (f) results of the IP-RAN, and (g) targeted photos.
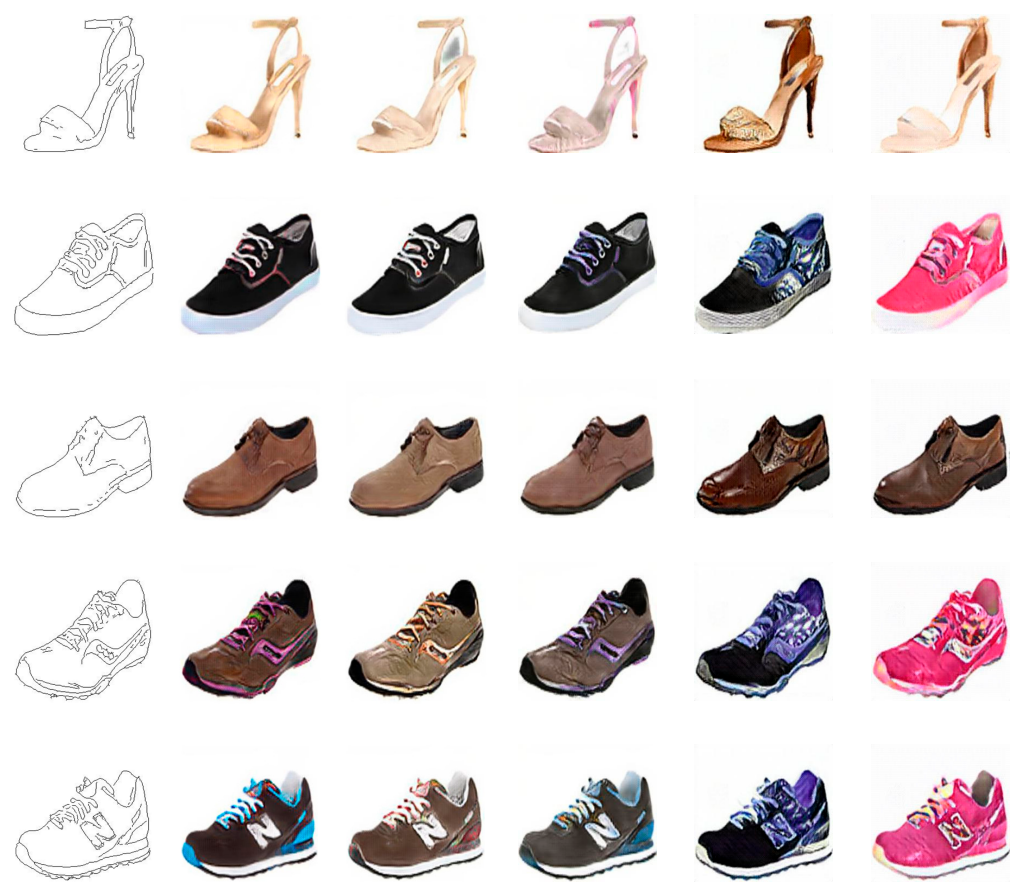

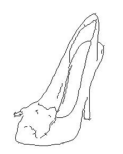

(a)

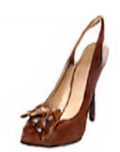

(b)

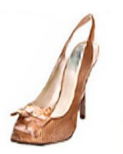

(c)

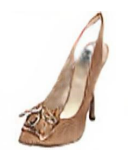

(d)

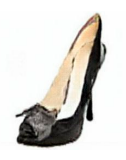

(e)

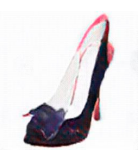

(f)

Figure 8. Samples result from edges to shoes. (a) input images, (b) results of the IP-RAN, (c) results of the pix2pix-cGAN, (d) results of PAN, (e) results of UTN-GAN, and (f) results of iPANs 
Table 4. Quantitative results of labels to architectural photos, bold results show good scores.

\begin{tabular}{cccccc}
\hline & PSNR(dB) & SSIM & UQI & VIF & FID \\
\hline Pix2Pix-cGAN & $\mathbf{1 3 . 3 7}$ & $\mathbf{0 . 2 5 5 9}$ & $\mathbf{0 . 8 1 9 5}$ & $\mathbf{0 . 0 5 4 1}$ & 113.53 \\
UTN-GAN & 12.78 & 0.2362 & 0.8016 & 0.0481 & 111.86 \\
PAN & 12.82 & 0.2370 & 0.8030 & 0.0477 & 112.47 \\
iPANs & 11.46 & 0.1765 & 0.7603 & 0.0382 & 140.70 \\
IP-RAN & 12.84 & 0.2426 & 0.8052 & 0.0488 & $\mathbf{1 1 0 . 2 9}$ \\
\hline
\end{tabular}

Table 5. Quantitative results of Edges to Shoes, bold results show good scores.

\begin{tabular}{cccccc}
\hline & PSNR(dB) & SSIM & UQI & VIF & FID \\
\hline Pix2Pix-cGAN & 19.33 & 0.7569 & $\mathbf{0 . 9 2 2 0}$ & 0.2092 & $\mathbf{5 9 . 9 3}$ \\
UTN-GAN & 15.41 & 0.6588 & 0.8255 & 0.1786 & 104.9 \\
PAN & 19.11 & 0.7389 & 0.9187 & 0.2034 & 62.13 \\
iPANs & 15.71 & 0.6671 & 0.8444 & 0.1778 & 117.1 \\
IP-RAN & $\mathbf{1 9 . 4 2}$ & $\mathbf{0 . 7 6 0 8}$ & 0.9179 & $\mathbf{0 . 2 1 5 3}$ & 62.15 \\
\hline
\end{tabular}

\subsubsection{Comparison with UTN-GAN, ID-CGAN and iPANs}

ID-CGAN and iPANs try to resolve the image de-raining problem. They aim to eliminate rain streaks from a given input rainy photos. Assuming un-predictable weather situations, the image de-raining or de-snowing alone is a challenging image-to-image conversion problem.

We try to resolve a single image de-raining task by the IP-RAN using a similar configuration to ID-CGAN. We train our adopted scheme on the image de-raining dataset provided by ID-CGAN [3]. This dataset contains 700 synthesizing images for training, whereas 100 artificial and 50 real-world rainy images are presented for testing purposes. Figure 9 shows the sample results of synthetic test images. As per the collection of ground-truth images are available against the set of synthetic test photos, we measure and report the quantitative outcomes in Table 6. Furthermore, we assess UTN-GAN, ID-CGAN, iPANs and IP-RAN on natural rainy images, and the results are shown in Figure 10.
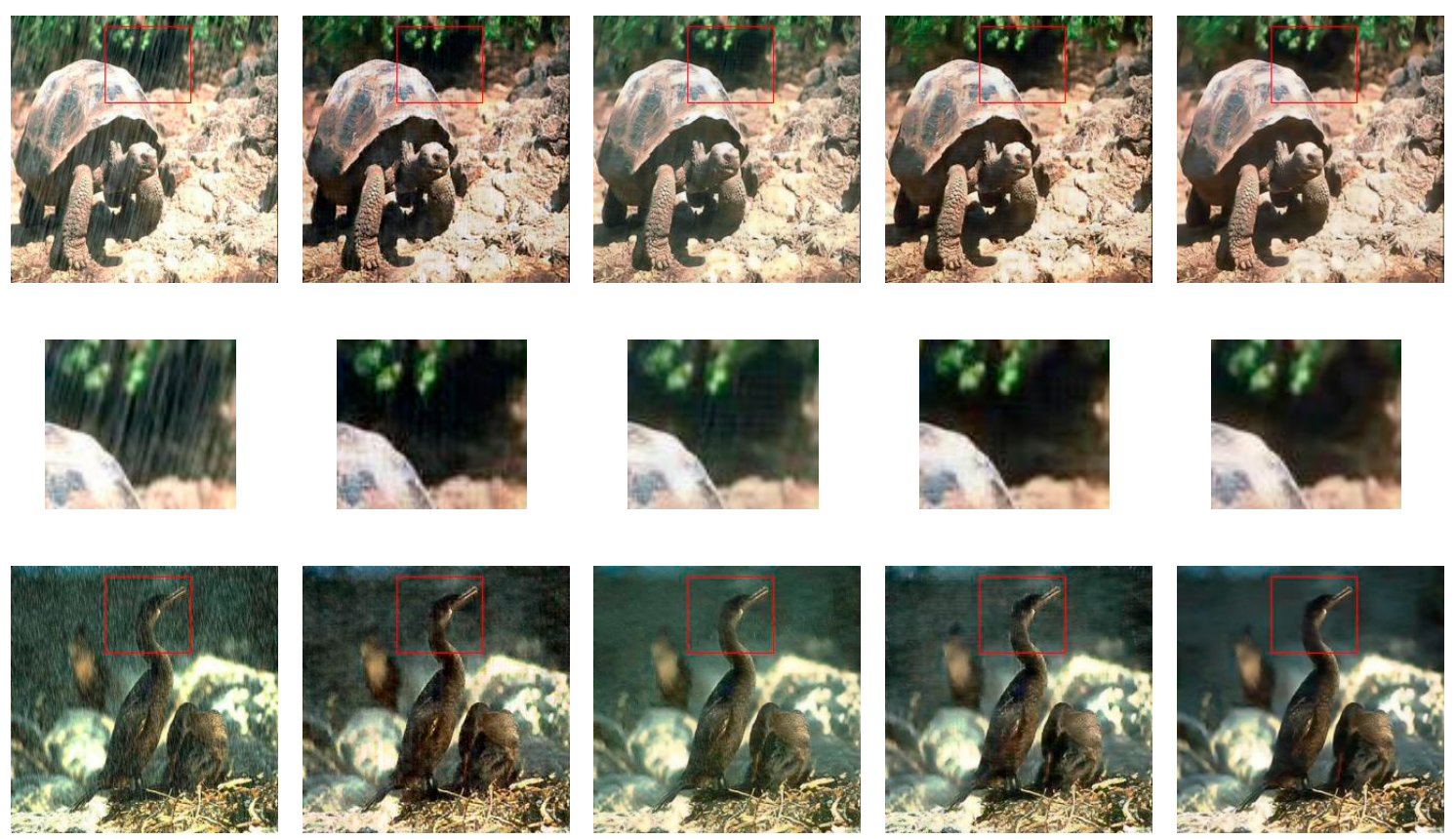

Figure 9. Cont. 

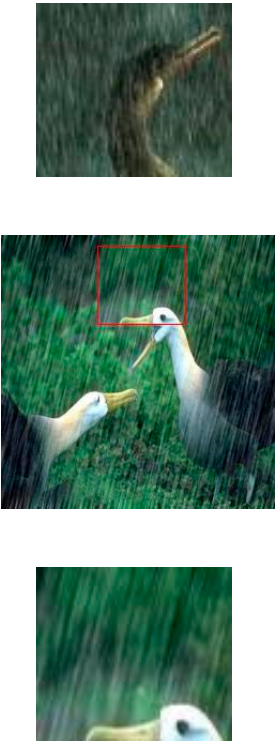

(a)
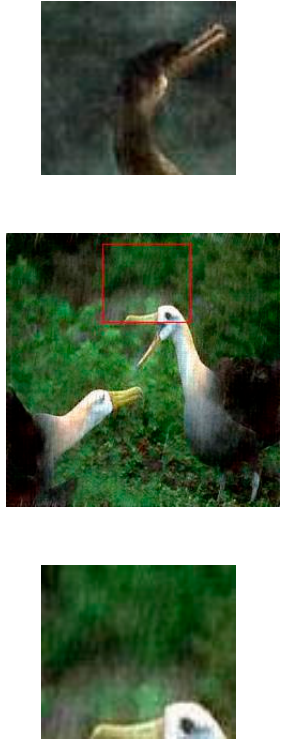

(b)
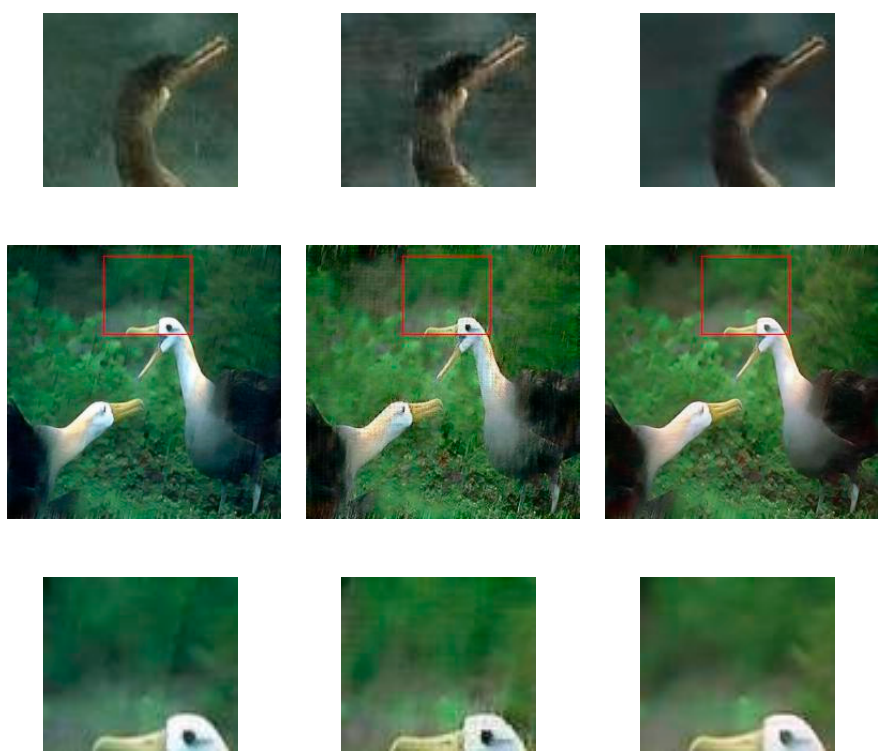

(c)

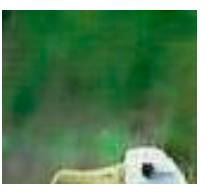

(d)

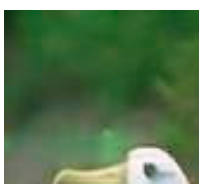

(e)

Figure 9. Sample results of synthetic test images. For good visual comparison, the smaller images below the test images represent specific regions-of-interest. (a) input images, (b) results of UTN-GAN, (c) results of ID-CGAN, (d) results of iPANs and (e) results of the IP-RAN.

Table 6. Quantitative results of image de-raining, bold results show good scores.

\begin{tabular}{cccccc}
\hline & PSNR(dB) & SSIM & UQI & VIF & FID \\
\hline UTN-GAN & 21.81 & 0.7325 & 0.9056 & 0.2939 & 127.4 \\
ID-CGAN & $\mathbf{2 4 . 4 2}$ & 0.8490 & $\mathbf{0 . 9 4 3 3}$ & 0.3708 & 76.71 \\
iPANs & 22.44 & 0.7687 & 0.9252 & 0.3101 & 112.72 \\
IP-RAN & 23.69 & $\mathbf{0 . 8 5 1 8}$ & 0.9412 & $\mathbf{0 . 3 7 4 0}$ & $\mathbf{7 5 . 9 0}$ \\
\hline
\end{tabular}

From Figures 9 and 10, we can observe that ID-CGAN, iPANs and the IP-RAN have accomplished great results in image de-raining tasks. The findings of the iPANs look slightly better, but contain some artifacts and blurriness. However, by examining the results carefully, the adopted scheme eliminates more rain-streaks with a lesser amount of color distortion. Moreover, as specified in Table 6, for a synthetic set of test images, the introduced method's evaluation scores and the resultant images are far more comparable with the corresponding ground-truth photos than with the results of the other methods. In the single image de-raining problem, the adopted method can accomplish more improved results than UTN-GAN, ID-CGAN, iPANs; one of the possible reasons is that these methods used skip-connection in their generator network. These skip-connection passes useful as well as unwanted information directly from the input image to the output images throughout the network and influence the results. Even though ID-CGAN achieved highest score in the PSNR and UQI metrics, still rain-streak can be seen in the resultant images of ID-CGAN. On the other hand, the adopted method tries to resolve the problem through the proposed loss function using an encoder-decoder generator structure. The novel training scheme of IP-RAN can benefit the generator to learn better-quality mapping from the input images to the output images, leading to improved performance. 

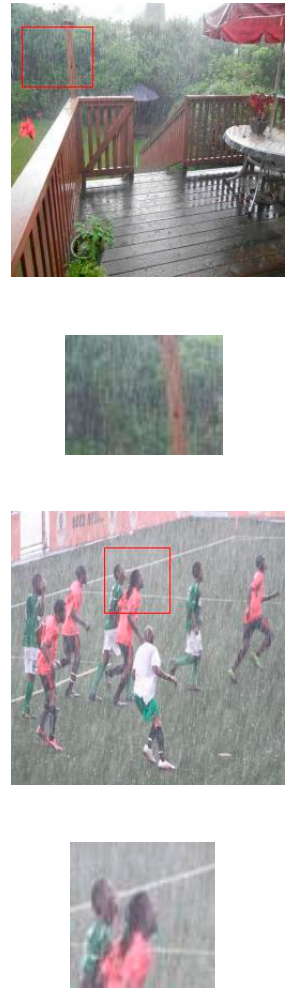

(a)
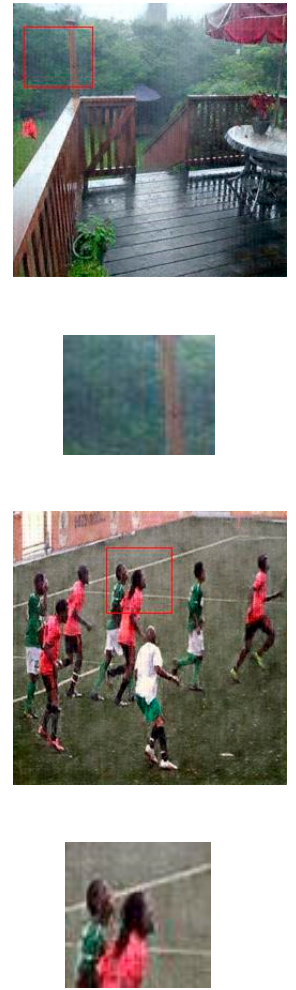

(b)
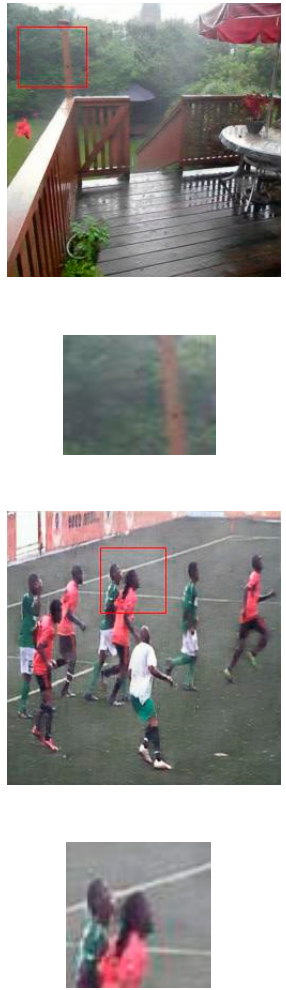

(c)
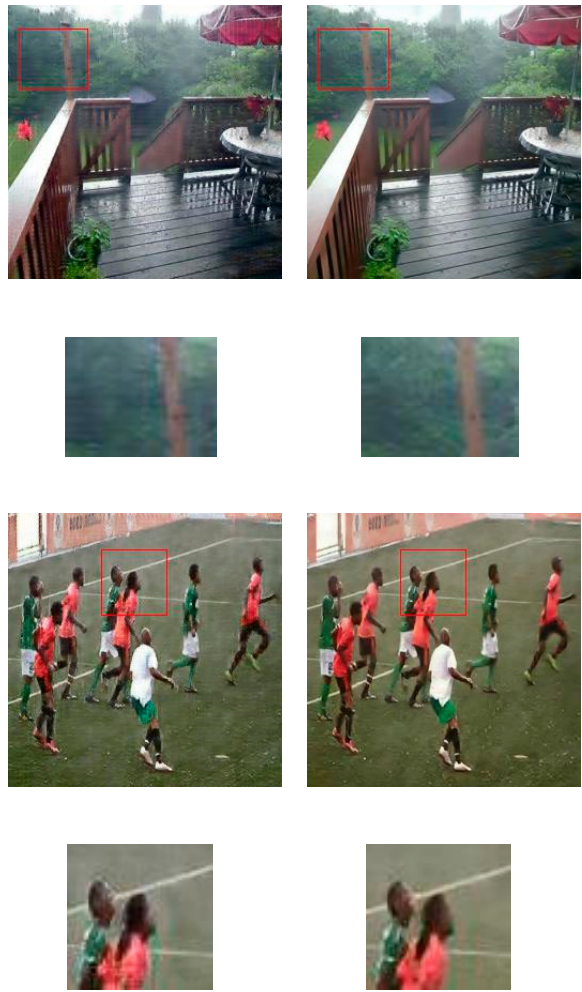

(d)

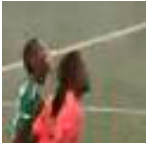

(e)

Figure 10. Sample results of real-world rainy images. For good visual comparison, the smaller images below the test images represent specific regions-of-interest. (a) input images, (b) results of UTN-GAN, (c) results of ID-CGAN, (d) results of iPANs and (e) results of the IP-RAN.

\section{Conclusions}

We have introduced a novel cGAN-based scheme to overcome the lack of information in input labels for imperfect paired datasets. In this work, we propose a novel Input-Perceptual and Reconstruction Adversarial Network (IP-RAN) for paired image-to-image conversion tasks as a general-purpose framework. We merge the input-perceptual loss with the adversarial and the per-pixel reconstruction Euclidean losses as an innovative loss function for imperfect paired datasets. Also, we analyze two popular generator configurations and evaluated their results quantitatively and qualitatively. A generator without skip-connections produced much better and visually pleasing results than a generator with skip-connections. We conducted extensive experiments on multiple datasets to assess the efficiency of the IP-RAN. The adopted scheme outperforms the state-of-the-art works for image-to-image conversion problems. The experimental results of several image-to-image conversion tasks illustrated that the proposed framework is efficient and capable of practical imperfect paired image-to-image conversion applications. In this study, we explored input-perceptual losses to feed the extra information of imperfect paired datasets for only paired image-to-image conversion tasks. Future work is required to examine the impact of input-perceptual losses for unpaired image-to-image conversion applications.

Author Contributions: Methodology, A.K. and W.J.; software and coding, A.K, and D.W.; Experimentation and formal analysis, A.K.; writing-original draft preparation, A.K.; writing-review and editing, A.K., M.A., R.A.N. and D.W.; supervision, W.J.; funding acquisition, W.J. All authors have read and agreed to the published version of the manuscript.

Funding: This research was funded by National Natural Science Foundation of China, grant number 61134002.

Conflicts of Interest: The authors declare no conflict of interest. 


\section{References}

1. Pathak, D.; Krahenbuhl, P.; Donahue, J.; Darrell, T.; Efros, A.A. Context encoders: Feature learning by inpainting. In Proceedings of the IEEE Conference on Computer Vision and Pattern Recognition, Las Vegas, NV, USA, 27-30 June 2016.

2. Bertalmio, M.; Sapiro, G.; Caselles, V.; Ballester, C. Image Inpainting. Available online: http://rbrad.ulbsibiu. ro/teaching/courses/docs/acs/bertalmi.pdf (accessed on 2 May 2020).

3. Zhang, H.; Sindagi, V.; Patel, V.M. Image de-raining using a conditional generative adversarial network. IEEE Trans. Circuits Syst. VideoTechnol. 2019. [CrossRef]

4. Ramwala, O.A.; Paunwala, C.N.; Paunwala, M.C. Image De-Raining for Driver Assistance Systems using U-Net based GAN. In Proceedings of the 2019 IEEE International Conference on Signal Processing, Information, Communication \& Systems (SPICSCON), Dhaka, Bangladesh, 28-30 November 2019.

5. Dong, C.; Loy, C.C.; He, K.; Tang, X. Image super-resolution using deep convolutional networks. IEEE Trans. Pattern Anal. Mach. Intell. 2015, 38, 295-307. [CrossRef] [PubMed]

6. Johnson, J.; Alahi, A.; Fei-Fei, L. Perceptual losses for real-time style transfer and super-resolution. arXiv 2016, arXiv:1603.08155.

7. Ledig, C.; Theis, L.; Huszár, F.; Caballero, J.; Cunningham, A.; Acosta, A.; Aitken, A.; Tejani, A.; Totz, J.; Wang, Z. Photo-Realistic Single Image Super-Resolution Using a Generative Adversarial Network. Available online: http://iie.fing.edu.uy/ \{\}mdelbra/DL2018/papers/11_2017_Ledig_CVPR.pdf (accessed on 3 May 2020).

8. Wang, T.-C.; Liu, M.-Y.; Zhu, J.-Y.; Tao, A.; Kautz, J.; Catanzaro, B. High-Resolution Image Synthesis and Semantic Manipulation with Conditional Gans. Available online: https:/davidwatkinsvalls.com/files/papers/ 1711.11585.pdf (accessed on 3 May 2020).

9. Nasrollahi, K.; Moeslund, T.B. Super-resolution: A comprehensive survey. Mach. Vis. Appl. 2014, 25, 1423-1468. [CrossRef]

10. Dai, Q.; Cheng, X.; Qiao, Y.; Zhang, Y. Crop Leaf Disease Image Super-Resolution and Identification with Dual Attention and Topology Fusion Generative Adversarial Network. IEEE Access 2020, 8, 55724-55735. [CrossRef]

11. Elad, M.; Aharon, M. Image Denoising via Sparse and Redundant Representations over Learned Dictionaries. IEEE Trans. Image Process. 2006, 15, 3736-3745. [CrossRef]

12. Kumar, R.; Maji, S.K. A Novel Framework for Denoised High Resolution Generative Adversarial Network-DHRGAN. In Proceedings of the 2020 7th International Conference on Signal Processing and Integrated Networks (SPIN), Noida, India, 27-28 February 2020.

13. Matsui, T.; Ikehara, M. GAN-Based Rain Noise Removal from Single-Image Considering Rain Composite Models. IEEE Access 2020, 8, 40892-40900. [CrossRef]

14. Gatys, L.A.; Ecker, A.S.; Bethge, M. Image Style Transfer Using Convolutional Neural Networks. Available online: http://liaoqing.me/course/AI\%20Project/[2016\%20CVPR]Image\%20Style\%20Transfer\% 20Using\%20Convolutional\%20Neural\%20Networks.pdf (accessed on 4 May 2020).

15. Khan, M.W. A survey: Image segmentation techniques. Int. J. Future Comput. Commun. 2014, 3, 89. [CrossRef]

16. Chen, X.; Duan, Y.; Houthooft, R.; Schulman, J.; Sutskever, I.; Abbeel, P. Infogan: Interpretable Representation Learning by Information Maximizing Generative Adversarial Nets. Available online: http://resources.dbgns. com/study/GAN/InfoGAN.pdf (accessed on 4 May 2020).

17. Zhang, R.; Isola, P.; Efros, A.A. Colorful Image Colorization. Available online: https://richzhang.github.io/ colorization/resources/colorful_eccv2016.pdf (accessed on 4 May 2020).

18. Chen, Y.; Lai, Y.-K.; Liu, Y.-J. Transforming Photos to Comics Using Convolutional Neural Networks. Available online: https://core.ac.uk/download/pdf/82967487.pdf (accessed on 4 May 2020).

19. Cheng, Z.; Yang, Q.; Sheng, B. Deep colorization. In Proceedings of the IEEE International Conference on Computer Vision, Santiago, Chile, 7-13 December 2015.

20. Long, J.; Shelhamer, E.; Darrell, T. Fully Convolutional Networks for Semantic Segmentation. Available online: https://computing.ece.vt.edu/ \{\}f15ece6504/slides/L13_FCN.pdf (accessed on 4 May 2020).

21. Goodfellow, I.; Pouget-Abadie, J.; Mirza, M.; Xu, B.; Warde-Farley, D.; Ozair, S.; Courville, A.; Bengio, Y. Generative Adversarial Nets. Available online: https://chyson.net/papers/Generative\%20Adversarial\% 20Nets.pdf (accessed on 4 May 2020). 
22. Cheng, K.; Tahir, R.; Eric, L.K.; Li, M. An analysis of generative adversarial networks and variants for image synthesis on MNIST dataset. Multimed. Tools Appl. 2020, 1-28. [CrossRef]

23. Isola, P.; Zhu, J.-Y.; Zhou, T.; Efros, A.A. Image-to-Image Translation with Conditional Adversarial Networks. Available online: https://gangw.web.illinois.edu/class/cs598/papers/CVPR17-img2img.pdf (accessed on 4 May 2020).

24. Chen, X.; Xu, C.; Yang, X.; Song, L.; Tao, D. Gated-gan: Adversarial gated networks for multi-collection style transfer. IEEE Trans. Image Process. 2018, 28, 546-560. [CrossRef]

25. Wang, C.; Xu, C.; Wang, C.; Tao, D. Perceptual Adversarial Networks for Image-to-Image Transformation. IEEE Trans. Image Process. 2018, 27, 4066-4079. [CrossRef] [PubMed]

26. Mirza, M.; Osindero, S. Conditional Generative Adversarial Nets. Available online: http://resources.dbgns. com/study/GAN/conditional_gan.pdf (accessed on 4 May 2020).

27. Kupyn, O.; Budzan, V.; Mykhailych, M.; Mishkin, D.; Matas, J. Deblurgan: Blind Motion Deblurring Using Conditional Adversarial Networks. Available online: http://www.gwylab.com/pdf/deblur-gan.pdf (accessed on 4 May 2020).

28. Regmi, K.; Borji, A. Cross-View Image Synthesis Using Conditional Gans. Available online: https://openaccess. thecvf.com/content_cvpr_2018/papers_backup/Regmi_Cross-View_Image_Synthesis_CVPR_2018_paper.pdf (accessed on 4 May 2020).

29. Ge, H.; Yao, Y.; Chen, Z.; Sun, L. Unsupervised transformation network based on GANs for target-domain oriented image translation. IEEE Access 2018, 6, 61342-61350. [CrossRef]

30. Wu, C.-H.; Horng, S.-J.; Lee, P.-Z. A new computation of shape moments via quadtree decomposition. Pattern Recognit. 2001, 34, 1319-1330. [CrossRef]

31. Ravuri, S.; Mohamed, S.; Rosca, M.; Vinyals, O. Learning implicit generative models with the method of learned moments. arXiv 2018, arXiv:1806.11006.

32. Santos, C.N.D.; Mroueh, Y.; Padhi, I.; Dognin, P. Learning Implicit Generative Models by Matching Perceptual Features. Available online: https://www.researchgate.net/profile/Cicero_Dos_Santos2/publication/332264118_ Learning_Implicit_Generative_Models_by_Matching_Perceptual_Features/links/5d1e0ff6a6fdcc2462c0cccb/ Learning-Implicit-Generative-Models-by-Matching-Perceptual-Features.pdf (accessed on 7 May 2020).

33. Simonyan, K.; Zisserman, A. Very deep convolutional networks for large-scale image recognition. arXiv 2014, arXiv:1409.1556.

34. Russakovsky, O.; Deng, J.; Su, H.; Krause, J.; Satheesh, S.; Ma, S.; Huang, Z.; Karpathy, A.; Khosla, A.; Bernstein, M. Imagenet large scale visual recognition challenge. Int. J Comput. Vis. 2015, 115, 211-252. [CrossRef]

35. Huh, M.; Agrawal, P.; Efros, A.A. What makes ImageNet good for transfer learning? arXiv 2016, arXiv:1608.08614.

36. Voulodimos, A.; Doulamis, N.; Doulamis, A.; Protopapadakis, E. Deep learning for computer vision: A brief review. Comput. Intell. Neurosci. 2018, 2018, 13. [CrossRef]

37. Eigen, D.; Fergus, R. Predicting Depth, Surface Normals and Semantic Labels with a Common Multi-Scale Convolutional Architecture. Available online: http://iie.fing.edu.uy/ \{\}mdelbra/DL2017/papers/09_2015_ Eigen_ICCV.pdf (accessed on 8 May 2020).

38. Farabet, C.; Couprie, C.; Najman, L.; LeCun, Y. Learning hierarchical features for scene labeling. IEEE Trans. Pattern Anal. Mach. Intell. 2012, 35, 1915-1929. [CrossRef]

39. Noh, H.; Hong, S.; Han, B. Learning Deconvolution Network for Semantic Segmentation. Available online: http://www-prima.imag.fr/Prima/jlc/Courses/2018/PRML/Noh_Learning_Deconvolution_Network_ ICCV_2015_paper.pdf (accessed on 8 May 2020).

40. Garcia-Garcia, A.; Orts-Escolano, S.; Oprea, S.; Villena-Martinez, V.; Garcia-Rodriguez, J. A review on deep learning techniques applied to semantic segmentation. arXiv 2017, arXiv:1704.06857.

41. Fu, X.; Huang, J.; Ding, X.; Liao, Y.; Paisley, J. Clearing the skies: A deep network architecture for single-image rain removal. IEEE Trans. Image Process. 2017, 26, 2944-2956. [CrossRef] [PubMed]

42. Fu, X.; Huang, J.; Zeng, D.; Huang, Y.; Ding, X.; Paisley, J. Removing Rain from Single Images via a Deep Detail Network. Available online: https:/xueyangfu.github.io/paper/2017/cvpr/cvpr2017.pdf (accessed on 8 May 2020).

43. Ružić, T.; Pižurica, A. Context-aware patch-based image inpainting using Markov random field modeling. IEEE Trans. Image Process. 2014, 24, 444-456. [CrossRef] [PubMed] 
44. Qin, C.; Chang, C.-C.; Chiu, Y.-P. A novel joint data-hiding and compression scheme based on SMVQ and image inpainting. IEEE Trans. Image Process. 2013, 23, 969-978. [CrossRef]

45. Eigen, D.; Puhrsch, C.; Fergus, R. Depth Map Prediction from a Single Image Using a Multi-Scale Deep Network. Available online: http://datascienceassn.org/sites/default/files/Depth\%20Map\%20Prediction\% 20from\%20a\%20Single\%20Image\%20using\%20a\%20Multi-Scale\%20Deep\%20Network.pdf (accessed on 9 May 2020).

46. Liu, F.; Shen, C.; Lin, G. Deep Convolutional Neural Fields for Depth Estimation from a Single Image. Available online: https://www.cv-foundation.org/openaccess/content_cvpr_2015/app/3B_078.pdf (accessed on 9 May 2020).

47. Berthelot, D.; Schumm, T.; Metz, L. Began: Boundary equilibrium generative adversarial networks. arXiv 2017, arXiv:1703.10717.

48. Radford, A.; Metz, L.; Chintala, S. Unsupervised representation learning with deep convolutional generative adversarial networks. arXiv 2015, arXiv:1511.06434.

49. Brock, A.; Lim, T.; Ritchie, J.M.; Weston, N. Neural photo editing with introspective adversarial networks. arXiv 2016, arXiv:1609.07093.

50. Reed, S.; Akata, Z.; Yan, X.; Logeswaran, L.; Schiele, B.; Lee, H. Generative adversarial text to image synthesis. arXiv 2016, arXiv:1605.05396.

51. Vondrick, C.; Pirsiavash, H.; Torralba, A. Generating Videos with Scene Dynamics. Available online: https://pdfs.semanticscholar.org/7188/6726f0a1b4075a7213499f8f25d7c9fb4143.pdf (accessed on 9 May 2020).

52. Wu, J.; Zhang, C.; Xue, T.; Freeman, B.; Tenenbaum, J. Learning a Probabilistic Latent Space of Object Shapes via 3d Generative-Adversarial Modeling. Available online: https:/core.ac.uk/download/pdf/141473151.pdf (accessed on 9 May 2020).

53. Lotter, W.; Kreiman, G.; Cox, D. Unsupervised learning of visual structure using predictive generative networks. arXiv 2015, arXiv:1511.06380.

54. Zhu, J.-Y.; Krähenbühl, P.; Shechtman, E.; Efros, A.A. Generative Visual Manipulation on the Natural Image Manifold. Available online: https://www.philkr.net/media/zhu2016generative.pdf (accessed on 9 May 2020).

55. Arjovsky, M.; Chintala, S.; Bottou, L. Wasserstein generative adversarial networks. In Proceedings of the International Conference on Machine Learning, Sydney, Australia, 6-11 August 2017.

56. Gulrajani, I.; Ahmed, F.; Arjovsky, M.; Dumoulin, V.; Courville, A.C. Improved Training of Wasserstein Gans. Available online: http://www.cs.utoronto.ca/ \{\}bonner/courses/2020s/csc2547/papers/adversarial/improvedtraining-of-WGANs,-gulrajani,-nips2017.pdf (accessed on 9 May 2020).

57. Zhao, J.; Mathieu, M.; LeCun, Y. Energy-based generative adversarial network. arXiv 2016, arXiv:1609.03126.

58. Karras, T.; Aila, T.; Laine, S.; Lehtinen, J. Progressive growing of gans for improved quality, stability, and variation. arXiv 2017, arXiv:1710.10196.

59. Miyato, T.; Kataoka, T.; Koyama, M.; Yoshida, Y. Spectral normalization for generative adversarial networks. arXiv 2018, arXiv:1802.05957.

60. Wang, C.; Xu, C.; Yao, X.; Tao, D. Evolutionary generative adversarial networks. IEEE Trans. Evol. Comput. 2019, 23, 921-934. [CrossRef]

61. Denton, E.L.; Chintala, S.; Fergus, R. Deep Generative Image Models Using a Laplacian Pyramid of Adversarial Networks. Available online: https://research.fb.com/wp-content/uploads/2016/11/deep-generative-imagemodels-using-a-laplacian-pyramid-of-adversarial-networks.pdf (accessed on 13 May 2020).

62. Li, J.; Liang, X.; Wei, Y.; Xu, T.; Feng, J.; Yan, S. Perceptual generative adversarial networks for small object detection. In Proceedings of the IEEE Conference on Computer Vision and Pattern Recognition, Honolulu, HI, USA, 21-26 July 2017.

63. Sung, T.L.; Lee, H.J. Image-to-Image Translation Using Identical-Pair Adversarial Networks. Appl. Sci. 2019, 9, 2668. [CrossRef]

64. Zhu, J.-Y.; Park, T.; Isola, P.; Efros, A.A. Unpaired Image-to-Image Translation Using Cycle-Consistent Adversarial Networks. Available online: https://pdfs.semanticscholar.org/c43d/954cf8133e6254499f3d68e45218067e4941.pdf (accessed on 13 May 2020).

65. Yi, Z.; Zhang, H.; Tan, P.; Gong, M. Dualgan: Unsupervised Dual Learning for Image-to-Image Translation. Available online: https://www.cs.sfu.ca/ \{\}haoz/pubs/yi_iccv17_dualGAN.pdf (accessed on 14 May 2020). 
66. Kim, T.; Cha, M.; Kim, H.; Lee, J.K.; Kim, J. Learning to Discover Cross-Domain Relations with Generative Adversarial Networks. Available online: http://axon.cs.byu.edu/Dan/673/papers/kim.pdf (accessed on 14 May 2020).

67. Chen, Q.; Koltun, V. Photographic Image Synthesis with Cascaded Refinement Networks. Available online: https://www.cqf.io/papers/Photographic_Image_Synthesis_ICCV2017.pdf (accessed on 15 May 2020).

68. He, K.; Zhang, X.; Ren, S.; Sun, J. Deep Residual Learning for Image Recognition. Available online: http://www.cs.sjtu.edu.cn/ \{\}shengbin/course/cg/Papers\%20for\%20Selection/Deep\%20Residual\% 20Learning\%20for\%20Image\%20Recognition.pdf (accessed on 15 May 2020).

69. Mao, X.; Li, Q.; Xie, H.; Lau, R.Y.; Wang, Z.; Paul Smolley, S. Least Squares Generative Adversarial Networks. Available online: https://www.researchgate.net/profile/Haoran_Xie/publication/322060458_Least_ Squares_Generative_Adversarial_Networks/links/5bfad008a6fdcc538819cf3e/Least-Squares-GenerativeAdversarial-Networks.pdf (accessed on 15 May 2020).

70. Ulyanov, D.; Vedaldi, A.; Lempitsky, V. Instance normalization: The missing ingredient for fast stylization. arXiv 2016, arXiv:1607.08022.

71. Li, C.; Wand, M. Precomputed Real-Time Texture Synthesis with Markovian Generative Adversarial Networks. Available online: https://arxiv.org/pdf/1604.04382v1.pdf (accessed on 15 May 2020).

72. Tyleček, R.; Šára, R. Spatial Pattern Templates for Recognition of Objects with Regular Structure. Available online: https://pdfs.semanticscholar.org/3edc/81db7c70d9123ea04829a98fc9fd62b29b1d.pdf (accessed on 16 May 2020).

73. Yu, A.; Grauman, K. Fine-Grained Visual Comparisons with Local Learning. Available online: https: //aronyu.io/vision/papers/cvpr14/aron-cvpr14.pdf (accessed on 16 May 2020).

74. Xie, S.; Tu, Z. Holistically-Nested Edge Detection. Available online: https://pages.ucsd.edu/ \{\}ztu/publication/ iccv15_hed.pdf (accessed on 16 May 2020).

75. Kingma, D.P.; Ba, J. Adam: A method for stochastic optimization. arXiv 2014, arXiv:1412.6980.

76. Wang, Z.; Bovik, A.C.; Sheikh, H.R.; Simoncelli, E.P. Image quality assessment: From error visibility to structural similarity. IEEE Trans. Image Process. 2004, 13, 600-612. [CrossRef]

77. Sheikh, H.R.; Bovik, A.C. Image information and visual quality. IEEE Trans. Image Process. 2006, 15, 430-444. [CrossRef]

78. Wang, Z.; Bovik, A.C. A universal image quality index. IEEE Signal Process. Lett. 2002, 9, 81-84. [CrossRef]

79. Heusel, M.; Ramsauer, H.; Unterthiner, T.; Nessler, B.; Hochreiter, S. Gans Trained by a Two Time-Scale Update Rule Converge to a Local Nash Equilibrium. Available online: http://papers.neurips.cc/paper/ 7240-gans-trained-by-a-two-time-scale-update-rule-converge-to-a-local-nash-equilibrium.pdf (accessed on 16 May 2020).

(C) 2020 by the authors. Licensee MDPI, Basel, Switzerland. This article is an open access article distributed under the terms and conditions of the Creative Commons Attribution (CC BY) license (http://creativecommons.org/licenses/by/4.0/). 